\title{
Study on the Control Strategy of Matrix Converter Motor System

\author{
shuping $\mathrm{Xu}$, erdong $\mathrm{Li}$ and xiaohui Su \\ School of Computer of Xi'an Technological University, Xi'an 710021 China \\ 563937848@qq.com,xusp686@163.com
}

\begin{abstract}
Keyword: Permanent magnet motor, Pole assignment, Direct torque control
\end{abstract}
\begin{abstract}
To overcome the defects of pure integrator initial value of the integral, the cumulative error and DC offset, permanent magnet synchronous motor stator flux observer method is proposed based on full-order state observer. The stator flux as state vector to construct the system state equation, the current as output vector to construct the system output equation, configuration observer poles proportional to the motor model pole, and use of the robust pole assignment algorithm in Matlab for calculate of observer feedback gain matrix. Experiments show that use of full-order state observer for the stator flux observed has higher accuracy, and it form of direct torque control system has better performance.
\end{abstract}

\section{Introduction}

The accuracy of flux observer directly affects the performance of the direct torque control system of permanent magnet synchronous motor, which is determined by the control principle of direct torque control [1]. The usual approach use a pure integrator, which has the advantage of being simple to implement and dependent on the motor parameters limited to the stator resistance. However, due to the influence of the integrator's initial value, error accumulation and non-ideal factors such as DC bias, it is difficult to realize the accurate observation of the stator flux by the pure integrator.

This paper is based on the method called induction motor flux linkage, which apply the modern control theory to the PMSM stator flux observation [2]. The PMSM stator flux full-dimensional state observer is designed using the robust pole assignment algorithm to estimate the stator flux linkage The results show that the full-dimensional state observer can overcome the defect of the pure integrator, and can be used to predict the performance of the direct torque control system using the stator flux-link state observer and the pure integrator.

However, what is known by the control theory is that the full-dimensional state observer is designed for linear time-invariant stationary systems and that motor parameters have the corresponding change with the motor running condition, the stator flux linkage error got by full dimension state observer designed on the basis of linear time invariant system could not be zero [3]. In order to overcome the time variation of the system and to realize the observation of the stator flux more accurately, the stator resistance which is easy to change in the process of motor operation is taken as variable parameter, and the Lyapunov stability theorem is used to mathematically deduce adaptive rate of resistance and a kind of all-digital fuzzy adaptive stator flux observation method. The simulation results show that the adaptive observer has higher accuracy for the stator flux observation and stronger robustness to the stator resistance variation than that of the PMSM stator flux observer

\section{Permanent Magnet Synchronous Motor Mathematical Model}

For the permanent magnet synchronous motor flux linkage voltage and current vector relationship ${ }^{[4]}$. The $\mathrm{d}$, q coordinate system is fixed on the rotating coordinate system of the rotor; the $\mathrm{x}, \mathrm{y}$ coordinate system is fixed on the rotating coordinate system of the stator. 
Taking the stator flux $\psi=\left\{\psi_{d}, \psi_{q}\right\}^{T}$ as the state vector and the stator current $i=\left\{i_{d}, i_{q}\right\}^{T}$ as the output vector $U=\left\{u_{d}, u_{q}, \psi_{f}\right\}^{T}$ as the input vector, the motor property model can be rewritten as a matrix:

$$
\frac{d}{d x}\left[\begin{array}{l}
\psi_{d} \\
\psi_{q}
\end{array}\right]=\left[\begin{array}{cc}
-\frac{R_{s}}{L_{d}} & \omega_{e} \\
-\omega_{e} & -\frac{R_{s}}{L_{q}}
\end{array}\right]\left[\begin{array}{l}
\psi_{d} \\
\psi_{q}
\end{array}\right]+\left[\begin{array}{lll}
1 & 0 & \frac{R_{s}}{L_{d}} \\
0 & 1 & 0
\end{array}\right]\left[\begin{array}{l}
u_{d} \\
u_{q} \\
\psi_{f}
\end{array}\right]\left[\begin{array}{l}
i_{d} \\
i_{q}
\end{array}\right]=\left[\begin{array}{cc}
\frac{1}{L_{d}} & 0 \\
0 & \frac{1}{L_{q}}
\end{array}\right]\left[\begin{array}{l}
\psi_{d} \\
\psi_{q}
\end{array}\right]+\left[\begin{array}{lll}
0 & 0 & -\frac{1}{L_{d}} \\
0 & 0 & 0
\end{array}\right]\left[\begin{array}{l}
u_{d} \\
u_{q} \\
\psi_{f}
\end{array}\right]
$$

Where - $d$, $q$ axis flux - $d$, $q$ axis voltage.

\section{Stator Flux Observations Based on Full - Dimensional State Observer}

Stator Flux Linkage Full-Dimensional State Observer Structure. In practice, not all of the state variables can be measured directly, so you can use the variables measured in the system to refactor state variables and artificially constructed a system that is same as the original system exactly. Because the system is man-made, the state variables of the system are fully measurable $[5,6]$.

Construction System:

$$
\left\{\begin{array}{l}
\dot{\hat{\boldsymbol{\psi}}}_{s}=\mathbf{A} \hat{\boldsymbol{\psi}}_{s}+\mathbf{B} \mathbf{u}_{s} \\
\hat{\mathbf{i}}_{s}=\mathbf{C} \hat{\boldsymbol{\psi}}_{s}+\mathbf{D} \mathbf{u}_{s}
\end{array}\right.
$$

Among them, $\hat{\boldsymbol{\Psi}}_{s}$ the state vector, the original system $\boldsymbol{\Psi}_{s}$ to be observed for the actual state vector estimates,

$$
\begin{aligned}
& \mathbf{A}=\left[\begin{array}{cc}
-\frac{R_{s}}{L_{d}} & \omega_{e} \\
-\omega_{e} & -\frac{R_{s}}{L_{q}}
\end{array}\right] \quad \mathbf{B}=\left[\begin{array}{ccc}
1 & 0 & \frac{R_{s}}{L_{d}} \\
0 & 1 & 0
\end{array}\right] \\
& \mathbf{C}=\left[\begin{array}{cc}
\frac{1}{L_{d}} & 0 \\
0 & \frac{1}{L_{q}}
\end{array}\right] \quad \mathbf{D}=\left[\begin{array}{ccc}
0 & 0 & -\frac{1}{L_{d}} \\
0 & 0 & 0
\end{array}\right] \\
& \hat{\boldsymbol{\Psi}}_{s}=\left[\begin{array}{ll}
\hat{\psi}_{s d} & \hat{\psi}_{s q}
\end{array}\right]^{T} \mathbf{u}_{s}=\left[\begin{array}{lll}
u_{s d} & u_{s q} & \psi_{f}
\end{array}\right]^{T} \mathbf{i}_{s}=\left[\begin{array}{ll}
i_{s d} & i_{s q}
\end{array}\right]^{T}
\end{aligned}
$$

From the above equation When $\boldsymbol{\psi}_{s}(\mathbf{0})=\hat{\boldsymbol{\psi}}_{s}(\mathbf{0})$ must have $\hat{\boldsymbol{\psi}}_{s}=\boldsymbol{\psi}_{s}$. But in general, it is impossible to guarantee that the initial conditions are identical at any time. Therefore, in order to eliminate the state error, error $\left(\boldsymbol{\psi}_{s}-\hat{\boldsymbol{\psi}}_{s}\right)$ feedback that is $\left(\mathbf{i}_{s}-\hat{\mathbf{i}}_{s}\right)$ feedback can be introduced on this basis of control theory. The advantage of introducing the feedback term is that when the initial state $\Psi_{s}$ and $\hat{\Psi}_{s}$ are biased, the deviation can not be diffused or oscillated with time. Second, the open-loop observer can not be adjusted when the matrix A changes. The deviations of $\boldsymbol{\Psi}_{s}$ and $\hat{\boldsymbol{\Psi}}_{s}$ are disadvantageous. The structure of the state observer is shown in the dashed box in Fig. 1. In the graph, $\mathrm{G}$ is the feedback gain matrix of the state observer. 


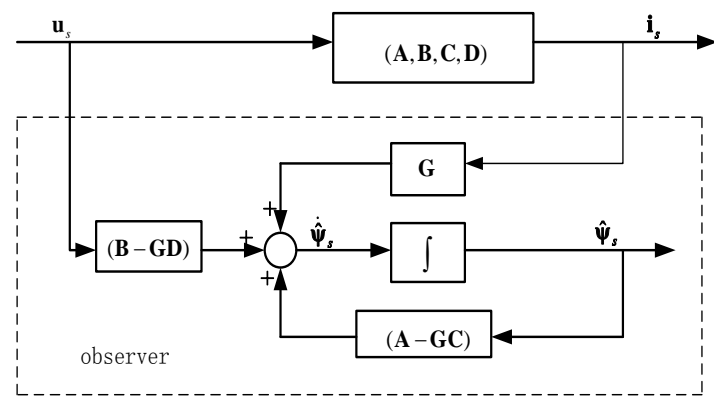

Figure 1. Block diagram of the whole-body state observer with stator flux linkage

From Fig. 1, the state estimation error of the observer is

$$
\boldsymbol{\psi}_{s}-\hat{\boldsymbol{\psi}}_{s}=\mathrm{e}^{(\mathbf{A}-\mathbf{G C}) t}\left[\boldsymbol{\psi}_{s}(0)-\hat{\boldsymbol{\psi}}_{s}(0)\right]
$$

Obviously, as long as the eigenvalues of the coefficient moments (A-GC) of the observer have negative real parts, the real value $\hat{\boldsymbol{\psi}}_{s}$ of the state estimation $\boldsymbol{\psi}_{s}$ asymptotic approximation state can be obtained.

Pole Configuration. The pole of the observer is the characteristic value of (A-GC), which is critical to the performance of the observer. Usually, the pole configuration of the observer should make the observer's response rate faster than the system model's, so that the observation error can be quickly converged [7, 8]. In order to determine the characteristic value of (A-GC), this paper adopts synchronous motor pole assignment method that configure the pole of the observer is proportion to the pole of the motor model.

Consider the poles of the motor, ie the eigenvalues of $|s \mathbf{I}-\mathbf{A}|=0$, that is, the eigenvalues of the following expressions:

$$
S^{2}+\left(\frac{R_{s}}{L_{d}}+\frac{R_{s}}{L_{q}}\right)_{S}+\frac{R_{s}}{L_{d}} \cdot \frac{R_{s}}{L_{q}}+\omega_{e}^{2}=0
$$

Two poles can be obtained:

$$
s_{1}=\frac{-\left(\frac{R_{s}}{L_{d}}+\frac{R_{s}}{L_{q}}\right)+\sqrt{\left(\frac{R_{s}}{L_{d}}+\frac{R_{s}}{L_{q}}\right)^{2}-4\left(\frac{R_{s}}{L_{d}} \cdot \frac{R_{s}}{L_{q}}+\omega_{e}^{2}\right)}}{2} s_{2}=\frac{-\left(\frac{R_{s}}{L_{d}}+\frac{R_{s}}{L_{q}}\right)-\sqrt{\left(\frac{R_{s}}{L_{d}}+\frac{R_{s}}{L_{q}}\right)^{2}-4\left(\frac{R_{s}}{L_{d}} \cdot \frac{R_{s}}{L_{q}}+\omega_{e}^{2}\right)}}{2}
$$

Set the pole of the observer to be $\mathrm{K}$ times the pole of the motor, as $o s_{1}=k \cdot s_{1} o s_{2}=k \cdot s_{2}$

\section{Simulation Analysis}

The direct torque control system of permanent magnet synchronous motor based on stator flux full-range state observer realized by Matlab / Simulink is shown in Fig. 2. Among them, the shadow-containing module is the full-dimensional state observer part of the stator flux. Motor parameters in Appendix A, the motor parameters I, speed setting 200r / min, the motor initial load is $2^{N} \cdot m, 0.2$ s sudden load to $3 N \cdot m$. In order to compare the effect of the initial position error of the flux linkage, the rotor flux initial position deviation angle of $\pi / 3 \mathrm{rad}$ is set for both observation methods in simulation. Then, Accuracy of Flux Observation Method and Its Influence on System Performance. 


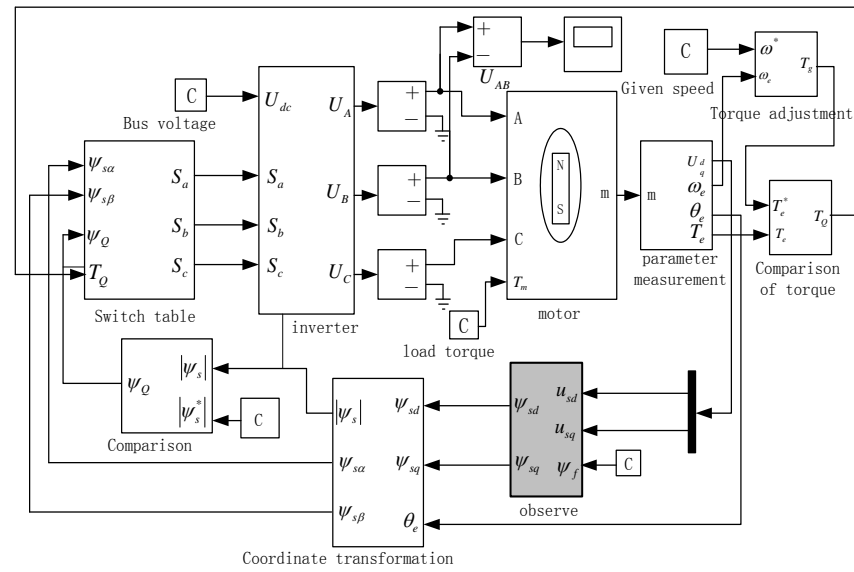

Figure 2. A block diagram of the direct torque control system based on the stator flux full-dimensional state observer.

Since the rotor has a constant initial magnetic field when estimating the stator flux linkage, the system needs to know the rotor's initial flux linkage to the stator. In the control system with the initial flux linkage inaccurate, the actual stator flux rotation path of the motor is not centered on the origin $\mathrm{O}$ but deviated from the origin. As can be seen from the flux component waveform shown in Fig. 3, pure integrator flux linkage $\alpha$ axis component waveform is asymmetric up and down and there is always a real magnetic flux DC bias, which will cause the motor torque and current fluctuations in the upper and lower. As shown in Fig. 4 (a) and Fig. 5 (a), the situation can not be observed in the real-time estimation of the flux to be suppressed. If the deviation is larger, the motor will not work properly. The stator flux trajectory obtained from the full-dimensional state observer is the origin $\mathrm{O}$, and the stator flux linkage obtained from the full-dimensional state observer has a large observation error at the start-up of the motor. Because the state observer uses a closed - loop flux linkage correction structure, the observation error is reduced quickly. Fig. 4 (b) and Fig. 5 (b) show the torque waveform and current waveform of the direct torque control system using the stator flux full-range state observer. The steady-state torque ripple and stator current harmonic components are good obviously than pure Integrator observation method.

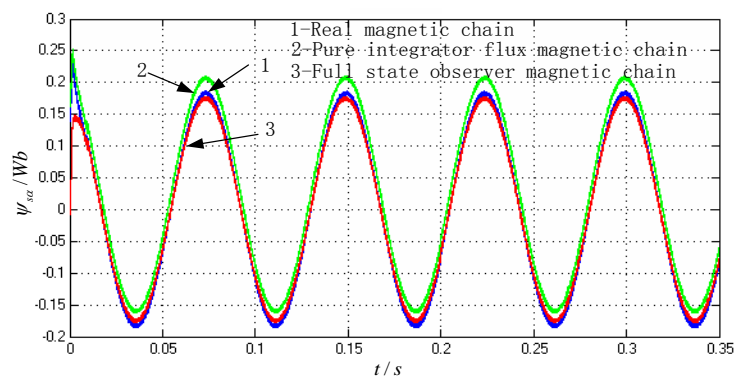

Figure 3. The stator flux component waveform

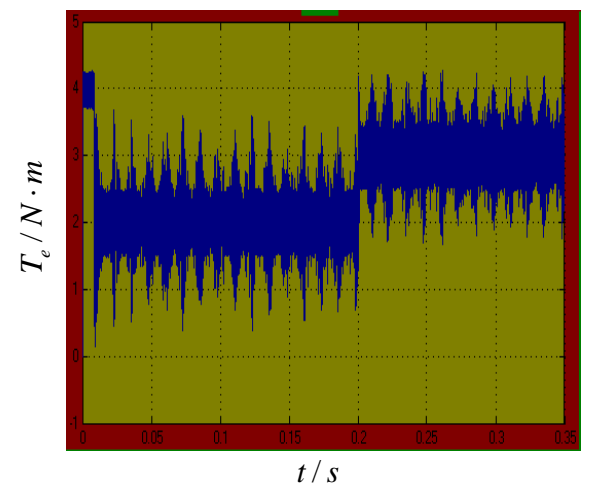


(A) Torque of a DTC system using a pure integrator

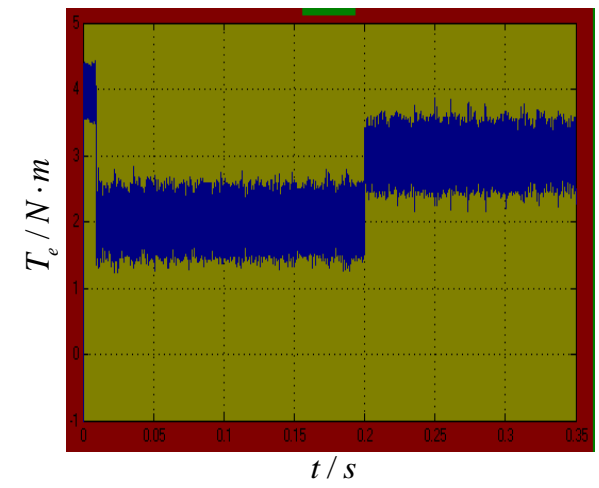

(B) Torque of the DTC system using the observer

Figure 4. Torque response of a DTC system with two flux linkage observations

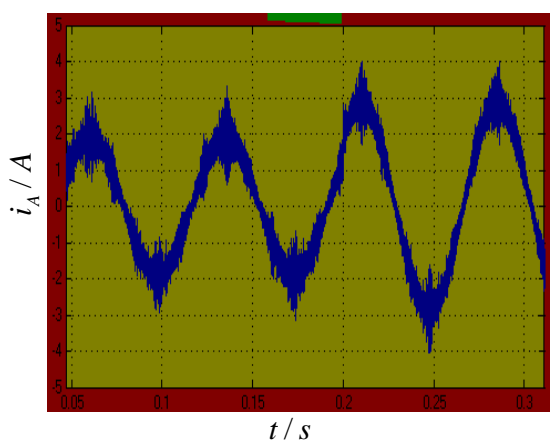

(A) Current in a DTC system using a pure integrator

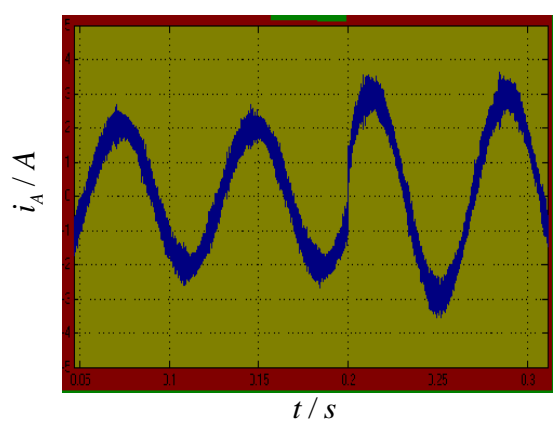

(B) The current of the DTC system using the observer

Figure 5. Shows the current response of the DTC system with two flux-linkage observations.

\section{Conclusion}

In order to overcome the shortcomings of the flux observer method of the conventional pure integrator, a PMSM stator flux observer method is presented in this paper, which is based on the robust pole assignment algorithm. Compared to the case where the initial flux of the rotor has some error, the pure flux integrator and the full-scale state observer are used as the stator flux trajectory and the $\alpha$ axial component waveform of the flux linkage obtained from the stator flux linkage observation. The results show that the stator flux linkage state observer can overcome the influence of the DC bias of the integrator on the observation effect, and can accurately observe the stator flux linkage. In addition, the permanent magnet synchronous motor (PMSM) is a time-varying system 
with parameter changing at runtime, and the full-dimensional state observer is designed for the linear time-invariant stationary system. Therefore, the further research direction is to apply the adaptive method to the stator magnetism. The stator flux linkage is used as the reference model and the stator flux linkage state equation with variable resistance is used as the adjustable model, which will deduce the stator resistance adaptive rate that will obtain the stator flux linkage adaptive observer to verify the experiment. The experimental results of stator flux observation by full - dimensional state observer and pure integrator show that the full - dimensional state observer with modern control theory has higher accuracy for stator flux observation and better system performance.

\section{Acknowledgment}

The authors wish to thank the cooperators. This research is partially funded by the Project funds in shaanxi province department of education (15JF019) and the Project funds in shaanxi province department of science industrial projects (2015GY067).

\section{References}

[1] T.Sebastian,G.R.Slemon,M.A.Rahman.Modeling of Permanent Magnet Synchronous Motors[J].IEEE Transactions on Magnetics,1986,22(9):1069-1086

[2] S.COLIN.Adaptive Speed Identification for Vector Control of Induction Motor without Rotational Transducers [J].IEEE Transactions on Industry Applications, 1992,28(5):1054-1061

[3] Hu Jun,Wu Bin.New Integration Algorithms for Estimating Motor Flux Over a Wide Speed Range[J].IEEE Transactions on Power Electronics, 1998,13(5):969-977

[4] Xu Junfeng,Xu Yinglei,Xu Jianping,et al.A New Control Method for Permanent Magnet Synchronous Machines with Observer[C].in Conf.Aachen Germany:35th IEEE Power Electronics Specialists Conference,2004:1404-1408

[5] Young Sam Kim,Sang Kyoon Kim, Young Ahn Kwon.MRAS Based Sensorless Control of Permanent Magnet Synchronous Motor[C]. In Conf.Fukui,Japan:SICE Annual Conference, 2003:1632-1637

[6] L.Tang,M.F.Rahman.A New Direct Torque Control Strategy for Flux and Torque Ripple Reduction for Induction Motors Drive by Space Vector Modulation[C].PESC2001:1440-1445

[7] Lixin Tang,Limin Zhong,Muhammed Fazlur Rahman,, et a1. A Novel Direct Torque Control for Interior Permanent-Magnet Synchronous Machine Drive with Low Ripple in Torque and Flux -A Speed-Sensorless Approach [J]. IEEE Transactions on Industry Applications,2003,39 (6): $1748-1756$

[8] Jun Zhang,M.F.Rahman,Direct Flux Vector Control Scheme for Induction Machine Drives with Space Vector Modulation[C]. IAS 2005 Conference, 1798-1804

\footnotetext{
About the Author:

Biography: Xu shuping, (1974-05-07), female (the Han nationality), Shaan'xi Province, Working in $\mathrm{Xi}$ 'an technological university, professor, the research area is computer control.
} 